Théologiques

Théologiques

\title{
Le démonique comme perversion du divin d'après Paul Tillich
}

\section{Jean Richard}

Volume 5, numéro 1, mars 1997

\section{Satan}

URI : https://id.erudit.org/iderudit/024944ar

DOI : https://doi.org/10.7202/024944ar

Aller au sommaire du numéro

\section{Éditeur(s)}

Faculté de théologie de l'Université de Montréal

\section{ISSN}

1188-7109 (imprimé)

1492-1413 (numérique)

Découvrir la revue

\section{Citer cet article}

Richard, J. (1997). Le démonique comme perversion du divin d'après Paul Tillich. Théologiques, 5(1), 89-113. https://doi.org/10.7202/024944ar

\section{Résumé de l'article}

Cette étude présente la conception du démonique chez Paul Tillich, plus spécialement à partir de sa monographie de 1926 sur " Le démonique ». Le parcours s'accomplit alors selon trois niveaux d'analyse : (1) une théorie du démonique, conçu comme une perversion du sacré, comme un surgissement inordonné des forces vives constituant le fondement de tout être; (2) une histoire des religions, conçues elles-mêmes comme des institutions antidémoniques; (3) une investigation des forces démoniques dominantes à l'époque présente. d'utilisation que vous pouvez consulter en ligne.

https://apropos.erudit.org/fr/usagers/politique-dutilisation/ 


\title{
Le démonique comme perversion du divin
d'après Paul Tillich
}

\author{
Jean RICHARD \\ Faculté de théologie \\ Université Laval
}

\section{RÉSUMÉ}

Cette étude présente la conception du démonique chez Paul Tillich, plus spécialement à partir de sa monographie de 1926 sur "Le démonique ". Le parcours s'accomplit alors selon trois niveaux d'analyse: (1) une théorie du démonique, conçu comme une perversion du sacré, comme un surgissement inordonné des forces vives constituant le fondement de tout être; (2) une histoire des teligions, conçues elles-mêmes comme des institutions antidémoniques; (3) une investigation des forces démoniques dominantes à l'époque présente.

This study presents Paul Tillich's conception of the demonic, in particular, through his 1926 monograph The Demonic. It will be led using three levels of analysis : (1) a theory of the demonic, understood as a perversion of the sacred, as an uprising of vivid forces which constitute the foundation of all being; (2) a history of religions understood as anti-demonic institutions; (3) an investigation of the demonic forces that are dominant in the present age.

Pouvons-nous, aujourd'hui encore, parler théologiquement du démon? Sommes-nous réduits à traiter de l'histoire des croyances au démon, en commençant par les croyances bibliques? Quand les théologiens signalent un retour du démon dans l'imaginaire collectif, ne s'agit-il pas encore des croyances? D'autres pourront bien insister: le démon existe vraiment; plusieurs faits terrifiants, qui surviennent tout autour de nous, le prouvent suffisamment. Mais ce genre de discours n'impressionnera probablement plus grand monde aujourd'hui, surtout pas "les esprits cultivés qui méprisent les démons", comme aurait dit Schleiermacher. Ceux-ci auront 
tôt fait de réduire ces phénomènes à des cas psychanalytiques ou psychiatriques.

La question théologique qui m'occupe se situe quelque part entre ces deux extrêmes : entre la simple question des figures de l'imaginaire et la question dramatique de l'existence des démons. C'est la question de la force symbolique d'un élément spécifique de l'univers religieux, le démon. Parler du démon a-t-il encore un sens aujourd'hui? Traduite en langage plus technique, cette question revient à dire: le symbole religieux "démon" a-t-il encore un sens actif, une force significative; peut-il être encore aujourd'hui un symbole vivant ${ }^{1}$ ?

Bien sûr, le travail théologique ne saurait réanimer un symbole religieux qui aurait perdu toute vitalité. Mais la théologie peut tabler sur les éléments toujours actifs d'un symbole. Il sera donc possible de suivre différentes directions. Dans le cas qui nous occupe, on pourrait, par exemple, partir du "retour du merveilleux », du " retour des anges et des démons », et y reconnaître un signe des temps : une recherche de sens en direction du surnaturel. Mais cette voie du surnaturel n'est pas celle que j'entends suivre ici. Je voudrais plutôt m'attaquer au noyau dur de notre culture occidentale, son fondement économique, pour y déceler les traces du démonique. Voilà pourquoi j'ai choisi la voie du Tillich allemand, qui débouche elle-même sur la théologie de la libération.

Je pense tout particulièrement ici à un texte bien connu, "Le démonique », paru sous forme de monographie à Tübingen en 1926, traduit aux États-Unis dix ans plus tard et, tout récemment, en français. Dès sa parution, cette étude attira l'attention. L'ancien professeur de Tillich, Fritz Medicus, en fut très impressionné; et lors d'un colloque en Suisse, au début de 1928, Tillich en discuta longuement et passionnément avec Max Scheler (PAUCK 1976: 108-109). Il en fut de même pour la version américaine. À l'occasion d'un dîner à la Maison Blanche, le viceprésident Henry Wallace s'adressa à lui : "Votre nom est Tillich? Vous devez être l'auteur de l'article sur le démonique. Je suis très heureux de vous rencontrer. C'est là une idée importante; j'aimerais la mieux comprendre » (PAUCK $1976:$ 205). Tillich lui-même, semble-t-il, plaçait ce texte en tête de liste de ses œuvres. Walter Braune raconte qu'il lui confia au cours d'une conversation: « $\mathrm{Si}$ l'on voulait brûler tous mes

1 Je pose donc ici, au sujet du démon, la même question qu'à propos de Dieu dans mon étude de 1994, "Dire Dieu aujourd'hui : conditions d'un discours signifiant », dans C. MÉnARD et F. VilleneUne (dir.) Dire Dieu aujourd'hui. Montréal, Fides (Héritage et projet 54), 15-46. 
écrits, on devrait au moins épargner celui sur le démonique » (ALBRECHT/SCHÜSSLER $1986: 71$ ).

Cela nous autorise à concentrer notre analyse sur cet écrit qui exprime au mieux la pensée de Tillich sur le démonique. On ne doit pas être dupe cependant, et croire que tout cela est sorti d'un seul jet. Plusieurs essais ont précédé ce grand texte. Tillich les mentionne dans un écrit antérieur de la même année 1926 :

Dans un article sur la théorie de l'État chez Augustin, j'ai montré dans cette revue que, sans le concept du démonique avec sa dialectique particulière, on ne peut comprendre la première attitude chrétienne face à l'État. Auparavant déjà, dans mes "Grandes lignes du socialisme religieux", j'avais placé ce concept au centre de la critique socio-éthique de l'époque; et dans ma présentation de la philosophie de la religion, qui vient de paraitre dans le « Manuel de philosophie » de Dessoir, le concept du démonique a une importance décisive autant pour l'essence que pour l'histoire de la religion. C'est ma conviction que ce concept est d'une importance non seulement historique, mais aussi dogmatique et avant tout éthique, et qu'il ne doit pas figurer comme "traité du démon "dans un appendice au traité du péché, mais qu'il doit plutôt être développé, à partir du fondement constitué par la philosophie de la religion, dans toutes les principales parties de la systématique (TILliCH 1926b: 155).

Retenons surtout trois choses dans ce texte. D'abord, la conviction que le concept du démonique touche au cœur même de la dogmatique, qu'il doit donc se retrouver dans toutes les parties de la théologie systématique. Tillich pense sans doute ici au cours de dogmatique qu'il a donné à Marbourg l'année précédente ${ }^{2}$. Un chercheur attentif a pu dire, en effet, que "dans l'ouvrage de 1925, les termes "démonique", "démonie ", "démonisation", à l'instar de ceux énonçant le divin, ne se limitent pas à une ou deux thèses dans l'ensemble de l'exposé de Tillich; les éléments de cette constellation apparaissent non seulement dans toutes les parties de l'ouvrage, mais ils en constituent en fait l'intrigue principale " (DUMAS 1997). J'en conclus pour ma part que les deux textes sur le démonique, publiés par Tillich en 1926, sont, non pas des extraits, mais des fruits directs de son cours de 1925.

2 Ce texte, que Tillich a tenté par deux fois de récrire pour publication, n'est paru finalement qu'en 1986, grâce aux soins de Werner SCHüSSLER : Dogmatik. Marburger Vorlesung von Tillich. La traduction française doit paraître au cours de 1997 dans la collection des « CEuvres de Paul Tillich. » 
Autre chose à noter : l'importance avant tout éthique de ce concept du démonique. Le contexte immédiat montre bien qu'il s'agit ici de l'éthique sociale, en rapport plus précisément avec la problématique du socialisme religieux. C'est dans le même sens qu'il faut comprendre la première phrase d'un article de 1919 sur le socialisme : "Jusqu'à présent c'était le problème dogmatique qui occupait l'Église; ce sera désormais le problème éthique " (TILLICH 1919b:13). Voilà bien une idée qui embraye directement sur la théologie de la libération.

Enfin, troisième remarque, d'ordre méthodologique cette fois. Tillich mentionne ici une triple perspective sur la question du démonique : une approche philosophique (Wesenlehre), une approche historique (Geistesgeschichte) et une approche systématique (dogmatique et surtout éthique). Cela correspond exactement aux trois niveaux des sciences de l'esprit dans Le système des sciences (TILLICH 1923a : 211-223). On peut aisément reconnaître cette triple approche dans la plupart des écrits de Tillich sur le démonique à cette époque, tout spécialement dans la monographie de 1926. Nous l'adopterons ici pour déterminer l'ordre de l'exposé.

\section{Les profondeurs du démonique}

La première démarche, d'ordre philosophique, vise à retracer les racines ontologiques du démonique. Une approche immédiatement théologique chercherait d'emblée à déterminer la relation entre Dieu et Satan. Tillich procède plutôt par la voie de l'ontologie: il cherche dans les profondeurs de l'être le rapport entre le divin et le démonique.

\subsection{Les représentations du démonique}

Cette enquête philosophique est pourtant tout autre chose qu'une pure spéculation. Elle s'appuie sur l'expérience humaine, tout spécialement celle dont témoignent "l'art des primitifs et des Asiatiques, leurs images des dieux et leurs fétiches, leurs arts décoratifs et leurs masques de danse. » On est déconcerté par leur caractère difforme : aux formes organiques "se joignent d'autres éléments qui font éclater toutes nos conceptions des réalités organiques. "On est tenté d'expliquer le phénomène par le caractère rudimentaire de cet art. Les difformités seraient à mettre au compte de l'inexpérience; elles seraient l'effet d'un art non évolué. Mais un tel jugement prend pour critère les normes de l'art classique, humaniste. Il s'agit alors de représenter les formes (végétales, animales, humaines) dans toute leur pureté, dans leur splendeur rationnelle, pour autant que la forme esthétique se veut l'expression la plus adéquate de l'essence idéale de l'être. 
Or tel n'est justement pas le point de vue de cet art "primitif». Ce n'est pas l'essence idéale, rationnelle, des choses qu'il cherche à exprimer, mais les forces vitales qui s'exercent, qui se manifestent en elles. L'art classique présente ces énergies vitales comme parfaitement contenues, maîtrisées par la forme rationnelle. Ici, au contraire, le flot déferle et brise toutes les formes existantes. Ainsi, "les mêmes forces vitales qui sous-tendent la forme vivante deviennent des principes destructeurs lorsqu'elles se font trop puissantes et se soustraient à l'ordre que la forme organique devrait leur imposer. " Tillich en conclut: "Il existe non seulement un défaut de forme, mais aussi une forme qui contrarie la forme; il existe non seulement des choses insuffisamment positives, mais aussi des choses opposées à ce qui est positif " (TILLICH 1926a: 125-126) Telle est précisément la réalité du démonique.

Le parallélisme est frappant avec la philosophie de la culture exposée dans une célèbre conférence de 1919. Tillich dit alors que les concepts y ont été élaborés à partir de l'expérience esthétique, plus précisément, sous l'influence de la peinture expressionniste. C'est de là surtout que vient l'idée d'une irruption de la substance religieuse, du contenu vital, dans la forme rationnelle: "Dans ces tableaux, un contenu (Gehalt) religieux faisant éclater la forme aspire à la forme... " Ce qu'on doit entendre au sens de la théonomie nouvelle, au sens d'un renouveau religieux où le divin ne s'impose plus d'en haut, de façon hétéronome, mais surgit de l'intérieur, des profondeurs de l'être, pour inonder et renouveler la forme autonome. Se manifeste alors cependant une certaine ambivalence : "Ainsi, dans cet art [expressionniste] s'exprime un "non" et un « oui » en profondeur; cependant, le "non ", l'élément destructeur de la forme, me paraît encore prépondérant, quoique ce ne soit pas l'intention des artistes, chez qui on retrouve une volonté passionnée pour un "oui" nouveau, absolu " (TILLICH 1919a: 40). Le démonique (destructeur) n'est donc pas encore ici clairement distingué du divin (créateur).

\subsection{L'effet destructeur du démonique}

L'ambiguiité sera levée avec les études sur le démonique. Marc Dumas constate alors une différence au niveau de la terminologie. Il note que Tillich réserve le mot "irruption" (Durchbrechen) pour le divin, tandis qu'il utilisera plutôt les termes « surgissement " (Hervorbrechen), «effraction" (Einbrechen) et " rupture "(Zerbrechen) à propos du démonique (DUMAS 1997). Car celui-ci se reconnaît précisément à son caractère destructeur : "Le démonique, c'est le surgissement, contraire à la forme, du fondement créateur des choses " (TILliCH 1926a : 129).

Voilà qui demande plus d'explication : comment un même fondement créateur peut-il apparaître tour à tour comme divin et comme démonique? 
On doit dire d'abord que la même substance religieuse originelle, source infinie de l'être, est à la fois fondement (Grund) et profondeur abyssale (Abgrund) de l'être. Cela signifie que le fondement comprend infiniment plus que ce qui est donné dans la forme d'un être particulier. Chaque être repose sur un abîme, pour autant qu'il puise son existence à une source inépuisable, qui le dépasse infiniment, qui pourrait donc l'envahir en le submergeant totalement. Car " nous ne devons pas concevoir l'inépuisabilité en question comme une inépuisabilité passive, comme un océan calme qu'aucun sujet, aucune forme ou aucun monde ne pourrait épuiser; nous devons plutôt la concevoir comme une inépuisabilité active, comme l'infinité intérieure et productive de l'être, c'est-à-dire comme le "feu dévorant " qui est véritablement la profondeur abyssale de toute forme " (TILLICH 1926a : 128-129).

Il y a donc deux modes possibles de surgissement des profondeurs sacrées de l'être : l'un est ordonné (c'est l'irruption du divin) et l'autre, désordonné (c'est la rupture du démonique). Dans un cas, la forme finie est comblée de la plénitude divine; dans l'autre, elle est détruite par l'effet du démonique. Quant au caractère ordonné ou désordonné du surgissement, il se détermine finalement d'après la forme inconditionnée (la vérité, la justice, etc.), selon qu'elle est affirmée ou récusée :

Le démonique peut assumer une telle qualité [sacrée], car il est une expression de l'abîme du sens; c'est pourquoi il possède, tout comme le sacré positif, des qualités extatiques. C'est une irruption destructrice, mais une irruption qui provient du même abîme que l'irruption de grâce. La différence est cependant que la grâce est une irruption dans la forme, qui reconnaît la forme, qui affirme la forme inconditionnée, alors que le démonique ne se soumet pas à la forme inconditionnée (TILLICH 1925a: 84).

Mais il faut pousser plus loin l'enquête et chercher la cause d'un tel surgissement désordonné, destructeur. Tillich indique alors « la tendance qui habite tout être et que l'on peut voir également à l'œuvre dans toute la nature : à savoir les forces originelles et vitales qui sont portées à transgresser toute forme pour se lancer dans l'illimité... " Plus concrètement, il s'agit des grandes pulsions vitales auxquelles se réfèrent, d'une façon ou d'une autre, philosophes et psychologues: "Sans prétendre proposer quelque chose de définitif, nous désignons l'instinct de puissance et l'instinct érotique comme les deux pôles et deux forces conjointes de l'inconscient; nous parvenons ainsi à mieux comprendre les forces démoniques mentionnées ci-dessus..." Les forces vitales de l'inconscient reçoivent par là une nouvelle signification, plus profonde, d'ordre ontologique. Et Tillich montre comment le processus d'approfondissement et d'universalisation de ces notions se trouve déjà amorcé dans les 
philosophies de la vie et dans la psychanalyse: «C'est de cette manière seulement que s'explique l'usage universel, et par conséquent impropre, de la volonté de puissance chez Nietzsche et de la sexualité chez Freud" (TILLICH 1926a : 130-132).

Nous avons parlé de l'abîme et de l'inépuisabilité au fond de tout être. Si nous adoptons maintenant le point de vue de l'individu (tout être existant) constitué par une forme déterminée, nous dirons que l'origine du démonique se manifeste dans sa volonté d'infini. C'est la volonté, inhérente à toute chose, de dépasser sa propre forme limitée pour embrasser l'infinité de l'être: "En toute chose demeure l'inépuisabilité interne de l'être, la volonté de réaliser en soi comme individu l'infinité active de l'être, la tendance à rompre sa propre forme limitée et le désir ardent de réaliser en soi la profondeur abyssale " (TILlICH 1926a: 129). Ainsi, "la contradiction de la revendication inconditionnelle de l'inconditionné " équivaut à " l'élévation du conditionné à l'inconditionnalité ». Tillich en conclut que "la possibilité du démonique repose sur le fait que [...] la créature veut s'emparer de l'inépuisable profondeur divine, qu'elle veut l'avoir pour elle-même en tant qu'individu; c'est par là que la puissance créatrice devient destructrice " (TILLICH 1926b: 156). L'idée du démonique comme perversion du divin prend ici tout son sens, à ce point précis où la notion du démonique rejoint celle de l'idolâtrie : la déification (Vergötterung) du fini.

\subsection{La possession démonique}

Il était question jusqu'ici de l'être individuel en général; considérons maintenant le cas de la personne humaine. Tel est bien le lieu propre du démonique : "Le démonique se réalise dans l'esprit [...]. Le démonique ne trouve d'abord toute son acuité que dans les formes spirituelles " (TILLICH 1926a : 130). En effet, l'esprit humain constitue la forme la plus élevée où peuvent s'exprimer les profondeurs abyssales de l'être. C'est à ce niveau qu'apparaît au mieux la tension entre les forces vitales et la forme rationnelle. La personne humaine possède la maîtrise de soi. La forme spirituelle domine, contrôle de façon consciente et libre les pulsions vitales issues du plus profond de l'être.

L'apparition du démonique sera là beaucoup plus manifeste, car le surgissement incontrôlé des forces sacrées de l'être se réalise alors dans l'esprit, le lieu de la liberté et de la maîtrise de soi. Il doit donc y avoir libre accueil, ouverture consciente à ces forces obscures. On peut même supposer que l'esprit y consent librement pour s'élever à l'infinité divine. Mais c'est tout le contraire qui se produit. Sous l'influence du démonique, la forme spirituelle est brisée, l'unité de l'esprit est rompue. L'esprit est envahi par une force étrangère, il est "possédé ": 
Le démonisme n'est pas une rechute à un niveau infraspirituel de l'être. L'esprit demeure esprit. Il demeure maître de lui-même face à la nature. Cependant quelque chose d'étranger prend possession de lui. Cette autre chose comprend les forces vitales; mais tout en étant spirituelle elle déforme l'esprit. C'est l'état de "possession" par lequel le démonisme se réalise dans la personne (TILLICH 1926a: 131).

Il ne s'agit donc pas d'une diminution mais d'une élévation, d'une exaltation incontrôlée de la conscience personnelle. La démythologisation que propose ici Tillich s'accomplit ainsi dans une direction bien particulière. Ce n'est pas la simple démythologisation sécularisante qui, dans les phénomènes de possession, ne voit rien d'autre que des maladies mentales. Ces maladies, comme toutes les autres, sont des défauts de la nature: on pourrait dire, des failles, des manques dans le processus d'actualisation des forces créatrices. Dans le cas du démonique, il s'agit, au contraire, du surgissement véhément de ces mêmes forces créatrices, mais dont le déferlement dans l'esprit produit un effet négatif, un effet d'aliénation. Cependant, la différence principale vient encore du fait que le phénomène se produit au niveau de l'esprit et, par conséquent, au niveau de la responsabilité humaine, avec le concours de la liberté.

On voit bien là tout le travail de réinterprétation, de démythologisation, que Tillich fait subir à la notion ancienne de "possession", qu'il assume pourtant dans sa théorie. En effet, la possession "démonique» dont il parle est bien différente de la possession «diabolique " dont il est question dans l'Écriture et dans l'histoire de la spiritualité chrétienne. Celle-ci se traite par l'" exorcisme", par un combat direct contre le (ou les) démon(s), un combat qui s'effectue comme par-dessus la tête du "possédé ", lui-même pure victime dans cette étrange affaire. On s'imagine mal que la possession démonique puisse se régler de cette façon. L'exorcisme n'a pas sa place ici ${ }^{3}$. Ce qui est requis, c'est un changement radical, une conversion profonde du cour, de l'état de possession à l'état de grâce.

Tillich ne parle donc pas des "possédés " au sens habituel du terme et sa théorie du démonique ne pourra être de grande utilité pour " traiter » ces cas. Mais de quoi s'agit-il alors? On l'entrevoit à travers la comparaison qu'il fait entre l'état de possession (Besessenheit) et l'état de grâce (Begnadenheit) :

3 Plus précisément, l'exorcisme est démythologisé en même temps que le démon. Il peut, par ailleurs, garder son sens originel, comme guérison (psychique), au même titre que les autres guérisons. 
Les états de possession et de grâce se correspondent. On peut être terrassé, inspiré, brisé par une force démonique ou divine : ce sont là des corrélats. Dans l'un et l'autre phénomène, ce sont les forces originelles et créatrices qui, brisant la forme, pénètrent dans la conscience. Dans les deux cas, l'esprit est élevé au-dessus de son isolement autonome; dans les deux cas il est assujetti à une puissance qui n'est pas une puissance naturelle, mais qui provient de la profondeur abyssale, laquelle supporte aussi la nature (TILLICH 1926a: 131).

L'état de grâce dont il est question ici est autre chose que l'état d'innocence. Il signifie un état de plénitude divine. C'est l'état d'une personne remplie de la force divine. On pourrait dire aussi bien que c'est le cas de la personnalité «charismatique». L'idée se retrouve dans le premier grand texte socialiste de Tillich, «La masse et l'esprit » (1922). Il distingue alors deux types de personnalité : la personnalité axée sur la forme rationnelle de la culture (la personnalité autonome) et la personnalité axée sur le contenu (Gehalt) profond de l'être et de la communauté (la personnalité théonome). Pour Tillich, seul ce deuxième type de personnalité peut maintenir le lien avec la masse, puisque les deux communiquent dans le même contenu. Tel est le cas, tout spécialement, de la personnalité du chef (TILLICH 1922 : 51-73).

Il semble bien que nous soyons sur la bonne piste. Nous en avons un indice quand Tillich affirme, dans la monographie de 1926, que la nature du démonique devient «le plus clairement visible, quand nous nous tournons de la personnalité vers la société, du démonisme psychique vers le démonisme social. " Dans le même sens, il dira ensuite: "L'objet de la destructuration démonique, c'est la personne se trouvant en contexte social et c'est aussi l'ordre social lui-même porté par elle " (TILlICH 1926a : 133-134).

On peut en conclure que l'état de possession qui caractérise la personnalité démonique n'est rien d'autre que l'inversion, la perversion, de l'état de grâce qui est celui de la personnalité charismatique. On pourrait proposer comme illustrations plusieurs figures de notre siècle : Adolf Hitler, Jim Jones, Luc Jouret, etc. Le mauvais génie du chef se transmet, avec sa force destructrice, à toute la communauté, nationale ou religieuse, qui subit son influence.

\subsection{Démonisme et péché}

Le développement qui suit, sur la relation entre le démonique et le péché, confirme encore ce qui vient d'être dit. Les deux ont ceci de commun qu'ils sont contraires à l'essence : par rapport à l'ordre positif des choses, ils marquent une aliénation. Dans la nouvelle conception de 
Tillich cependant, la relation est beaucoup plus étroite que ce que nous sommes habitués de penser. D'une part, le démonique communique avec le péché pour autant qu'il s'installe au cœur même de la personnalité, de la liberté humaine. D'autre part, la conception du péché s'approfondit, jusqu'à une dimension sacrée négative, celle même du démonique. Voyons tour à tour ces deux aspects.

Le démonique comporte nécessité et liberté, inévitabilité et responsabilité. Puisqu'il se réalise au cœur même de la personnalité, on ne peut le concevoir comme un phénomène qui s'imposerait de force à l'esprit humain. Il inclut toujours une part de responsabilité; il ne peut jamais être sans péché.

Par ailleurs, sans la dimension du démonique, on reste avec une notion purement moraliste (rationaliste) du péché. Ce n'est plus qu'une imperfection, un manquement, une faute, la transgression d'une loi, la désobéissance à un commandement. Et quand se manifeste le caractère extrinsèque du commandement, quand tombe la loi instituée, le péché disparaît avec elle. La notion du démonique peut donc aider à retrouver le sens du péché, en faisant redécouvrir sa profondeur religieuse. Il s'agit alors de tout autre chose que de la simple inobservance d'une loi. La contrariété de l'essence qui définit le péché signifie la rupture de la forme spirituelle et l'ouverture aux forces obscures de l'être qui, désormais sans contrôle, envahissent la personne. Ces mêmes forces vitales, créatrices, deviennent alors destructrices, sources d'aliénation. Dans la tradition chrétienne d'orientation paulinienne, la doctrine du péché originel rend bien cette idée d'une puissance de péché qui finit par dominer l'esprit quand elle n'est plus retenue, quand on lui laisse libre cours. La notion d'un péché collectif prend elle-même tout son sens, quand on reconnaît, à la source de l'être, la présence de pouvoirs transcendants, dont la signification peut être positive ou négative selon l'orientation qui leur est donnée. Un paragraphe de cette section du texte résume fort bien tout cela :

L'existence du péché collectif transporte au-delà de la liberté de l'individu, dans la couche préconsciente de la nature et dans l'ordre suprapersonnel de la communauté. Ce qui était signifié dans la doctrine du péché originel ne peut être véritablement compris sans le concept du démonique. L'élément de nécessité que comporte le péché, le paradoxe que, dans l'acte contraire à l'essence, responsabilité et inévitabilité sont liées correspond tout à fait à la dialectique du démonique. Car la caractéristique du démonique tient au fait qu'il pénètre dans la profondeur de ce qui est naturel et infrapersonnel d'une part, de ce qui est social et suprapersonnel d'autre part, et qu'il trouve cependant dans le centre de l'être personnel le lieu de sa 
réalisation. La vision du démonique surmonte la notion moraliste du péché. Ce n'est pas par hasard que l'Aufklärung, avec son combat contre la mythologie du démonique, qui était par ailleurs tout à fait justifié, perdit non seulement le concept du démonique, mais encore le concept religieux du péché (TILLICH 1926a: 135).

Sous un autre aspect encore, la notion du péché s'approfondit au contact de celle du démonique: dans sa relation à Dieu. Le péché implique toujours un certain rapport à Dieu. Ce peut être un rapport purement extérieur si Dieu est considéré simplement comme celui qui commande, qui récompense et qui juge. Mais la conscience démonique surgit elle-même avec le désir d'accaparer la puissance infinie de l'être: "C'est la volonté créatrice d'être comme Dieu qui conduit à la chute, et non pas le simple fait d'être vaincu par la nature sensible. " La puissance créatrice de l'être apparaît alors sous son aspect destructeur. Le visage de Dieu est changé, perverti : "la méfiance face à Dieu est la démonisation de Dieu dans la conscience humaine. L'homme n'ose pas s'abandonner à l'inconditionné parce qu'il le considère comme ce qui le juge, le détruit et le brise. " On voit bien, encore une fois, que l'exorcisme n'est pas la solution au problème. Seule la grâce peut aider: "Hors de la grâce, Dieu est loi, jugement, qui conduit au désespoir. Par le fait de la grâce, il devient Dieu, en opposition au démon. Voilà la relation la plus profonde entre le péché et le démonisme » (TILLICH 1926a : 135-136).

\section{Le sens religieux de l'histoire : un combat contre le démonique}

Nous avons, jusqu'ici, considéré de façon abstraite la dialectique du divin et du démonique. Avec Tillich, nous passons maintenant à l'approche historique. L'histoire religieuse de l'humanité, l'histoire des religions, prend ainsi tout son sens : elle apparaît comme « le combat du divin contre le démonique $»$. Or cette histoire religieuse, l'histoire du salut, ne se déroule pas de façon parallèle à l'histoire profane. Ici comme ailleurs, Tillich refuse la pensée supranaturaliste: " Ce caractère d'histoire du salut se trouve dans la profondeur de l'histoire. » Il s'agit donc d'une interprétation de l'histoire en profondeur, une interprétation qui tient compte du facteur démonique. Elle s'oppose ainsi à des interprétations trop naïvement optimistes, comme la conception utopique et la conception progressiste de l'histoire. Mais elle diffère tout autant de l'interprétation conservatrice. Car celle-ci "cherche finalement à se soustraire à l'attaque qui, à partir de l'éternel, est dirigée et doit être dirigée contre toute situation historique, parce qu'aucune forme, si ancienne et sacrée soit-elle, ne peut échapper à la démonisation " (TILLICH 1926a: 137). 
L'histoire des religions n'est donc pas simplement une histoire particulière en plus des autres, une histoire parallèle aux autres : à côté de l'histoire des arts et des sciences, à côté de l'histoire économique et politique. C'est plutôt le témoin du sens profond de l'histoire. Et elle se déroule sous le signe d'un combat contre le démonique, qui aboutit à la victoire finale du Christ : "De là provient une forte lumière sur l'histoire de la religion et la place du Christ dans cette histoire. Pour le message chrétien aux païens, l'affirmation que dans le Christ les forces démoniques sont vaincues était d'une importance fondamentale. En cela se trouve une conception déterminée de l'histoire de la religion "(TILLICH $1926 \mathrm{~b}: 158)$.

\subsection{L'arrière-fond démonique du sacré}

Il s'agit, bien sûr, d'une histoire théologique des religions. Cela a au moins l'avantage de mettre au clair, dès le départ, les présupposés théologiques de la synthèse proposée. Dans toute histoire des religions d'ailleurs, le point de départ est construit autant qu'intuitionné. Cela, non pas simplement faute de documents historiques concernant les origines. Car le point de départ est toujours déterminé en fonction de la perspective adoptée. Il s'agit le plus souvent du point de vue de la sécularité moderne : on imagine une origine purement profane. En ce temps-là, dit-on, les humains n'avaient d'autre préoccupation que d'assurer leur subsistance quotidienne; leurs moyens étant très réduits, ils se tournaient naturellement vers le ciel pour suppléer à leurs manques. Cette même perspective profane peut aussi s'orienter dans un sens plus rationaliste que pragmatique : les humains cherchaient à comprendre l'univers; ils inventèrent les dieux pour répondre à leurs questions.

Le point de départ de Tillich est tout différent. Sous l'influence de l'École d'histoire des religions, et plus encore, sans doute, sous l'influence de son ami Rudolph Otto, il suppose à l'origine le sens du sacré : tout baigne alors dans le sacré. Cela ne signifie pas seulement qu'il n'y a pas encore de nette distinction entre le sacré et le profane. Cela implique aussi que les différentes sphères de la culture n'ont pas émergé de l'univers mythique et sacré où elles sont encore contenues comme en germe. Il s'ensuit que, dans la sphère théorique, le vrai n'est pas apparu dans sa teneur rationnelle comme distinct et critique du faux. De même, dans la sphère pratique, la justice ne s'est pas encore manifestée avec ses exigences critiques face à l'ordre établi. Dans cet univers primitif, le vrai n'est donc pas séparé du faux, ni le juste de l'injuste. Ce qui fait dire à Tillich que "la qualité sacrée qui est attribuée à la plupart des choses et des événements, et même à beaucoup de leurs parties, confère à tout un caractère démonique aussi bien que divin. Ce qui est formé et ce qui est 
contraire à la forme, ce qui a un sens et ce qui est contraire au sens est apprécié de la même manière comme sacré " (TILliCH 1926a: 138). Il sera utile de lire ici un passage de la Philosophie de la religion, qui résume fort bien ce qui vient d'être dit :

Comme point de départ, l'unité de la forme et de la substance (Gehalt) est indifférente à l'égard de la division du sacré en divin et en démonique, tandis que, comme point d'arrivée, elle a éliminé le démonique en le subordonnant au divin. C'est donc la lutte du divin contre le démonique qui est à l'origine du mouvement entre le point de départ et le point d'arrivée. Naturellement, il faut entendre point de départ et point d'arrivée de manière simplement idéale, non pas de façon chronologique. Ce sont des constructions et non des réalités. La réalité se trouve entre ces deux points, mais d'une manière telle qu'elle est toujours poussée d'un point à un autre par un progrès ou un recul (TILliCH 1925a : 87).

\subsection{Les religions et le démonique}

La première institution émergeant de cet état de confusion primitive serait alors la religion elle-même. Celle-ci est requise précisément pour gérer le sacré, pour le contenir, pour éviter que ses débordements détruisent la société. Loin d'être une invention du diable, la religion, dans cette perspective, est instituée pour contrôler et réprimer le démonique.

Elle le fait d'abord en circonscrivant la zone du sacré. Outre cette distinction du sacré et du profane, elle instaure aussi la différence des sphères de la culture, en identifiant chacune par une divinité spéciale. Mais cela ne suffit pas : "la façon dont ces divers domaines de la culture sont reliés entre eux reste encore ici problématique et contraire au sens. Les uns contre les autres, ils demeurent isolés, fortuits et par conséquent démoniques » (TILliCH 1926a : 138).

L'avènement des grandes religions monothéistes marque sans doute un progrès quant à la conception d'un monde ordonné et unifié. Mais là non plus l'élément démonique n'est pas supprimé. Car le Dieu suprême du monothéisme repose lui-même sur un fondement limité, fini. C'est le Dieu d'une nation déterminée qui prétend à l'absoluité, à l'inconditionnalité. Alors, il «se brise avec sa nation dans les effets destructeurs de sa force démonique 》 (TILLICH 1926a : 138).

C'est dans le dualisme radical qu'apparaît au mieux le combat des religions. Car dans ces religions dualistes, " tous les éléments démoniques sont concentrés dans une divinité et tous les éléments divins dans l'autre; les deux se trouvent face à face avec une puissance égale. "Au terme de cet affrontement, le Dieu de la lumière apparaît comme le vainqueur 
final; il est reconnu comme le vrai Dieu. Ainsi, "la puissance supérieure du divin est maintenue. Mais cette puissance supérieure n'est pas une puissance inconditionnelle. Voilà pourquoi le dualisme n'est pas la victoire sur le démonique, et il ne peut l'être, parce que son Dieu de la lumière porte lui-même encore des traits démoniques : la lumière n'est pas le symbole du sens inconditionnel, de la forme et de l'unité spirituelle parfaites, mais elle est le symbole d'une sphère naturelle de l'être qui se tient devant une autre sphère naturelle " (TILLICH 1926a : 139).

La liste pourrait se poursuivre jusqu'à nos jours. On pourrait passer en revue les multiples figures des religions et y reconnaître toujours quelques traits démoniques, en dépit de leur intention profonde de combattre toutes les formes de démonisme. Bien plus, on pourrait conclure que c'est précisément sur le fond sacré des religions que les traits spécifiques du démonique apparaissent le plus manifestement, s'il est vrai que le démonique n'est rien d'autre qu'une perversion du divin et du religieux.

Il était donc à prévoir que la lutte contre le démonique finisse par prendre l'aspect d'une lutte contre les religions. Tillich connaît bien les antithèses de la religion et de la foi, de la religion et de la révélation, de la religion et de l'Évangile. Il ne les récuse pas, mais il va les intégrer elles-mêmes dans la dialectique de l'histoire des religions, pour montrer que toute religion comporte un élément autocritique, antireligieux, qui exprime son intention profonde, antidémonique.

Un premier type de protestation antidémonique et antireligieuse est donné dans l'ascétisme mystique, tel qu'illustré spécialement par les spiritualités orientales brahmanique et bouddhiste. La conscience cherche à se libérer du démonique par le renoncement: "Le "non" radical à toutes les formes de l'être supprime également le fondement démonique de tous les êtres." Mais la victoire sur le démonique signifie alors une victoire sur l'existence, elle-même éprouvée essentiellement comme démonique (TILliCH 1926a : 140).

Dans l'histoire de la religion chrétienne-occidentale, l'aspect critique antidémonique est déterminé par la prophétie juive. C'est là que "se dégagent toutes les positions de combat essentielles contre les forces démoniques. " La forme spirituelle de la vérité et de la justice s'y trouve affirmée comme forme divine : "Toute la sphère sacrée, qui se tient en dehors du parfait idéal éthique et social, est remise en question et, pour autant qu'elle se présente comme indépendante, elle est combattue en tant que démonique. "Il ne s'agit pas cependant d'un idéal abstrait, car la forme spirituelle est portée par l'unique vrai Dieu. Le monothéisme prend alors un caractère particulier : il est exclusif plutôt que monarchique. En effet, le Dieu des prophètes n'est pas simplement le premier d'une série, le 
plus haut placé dans la pyramide des dieux. Il est le Dieu "jaloux " qui exclut toute autre divinité, car il revendique l'inconditionnalité. Par contre, ce Dieu infini n'exclut pas la finitude du monde: "L'étant est créature et comme tel il n'est pas démonique. La contrariété du sens, la destruction de la forme, provient de la volonté de la créature et non pas d'un démon créateur » (TILliCH 1926a : 140-141).

Mais la religion des prophètes se distingue de la mystique orientale en ceci précisément que "le Dieu exclusif repose lui-même sur une base particulière, de nature nationale et culturelle. " D'où le danger d'un retour du démonique et le besoin d'un nouvel élément antidémonique : "Pour autant que ce Dieu élève alors une prétention exclusive, il doit également se l'appliquer à lui-même précisément dans cette particularité " (TILLICH 1926a : 141). Telle est pour Tillich le sens d'une troisième voie de salut, qu'il décrit en ces termes :

Une troisième voie pour triompher du démonique s'engage sur le terrain de la religion cultuelle elle-même. On peut la désigner comme la voie des mystères. Il est essentiel à l'esprit de cette voie que, de plein gré, le Dieu tourne contre lui-même la destruction démonique et que par là il en triomphe. Le mythe du Dieu souffrant et mourant, s'abaissant et devenant homme, est l'expression de cette voie. Le démonique se brise en lui, car la divinité porte elle-même la destruction. Le divin apparaît comme individu, mais de telle sorte qu'il se soumet au jugement dans son individualité même. Le divin est concrètement présent, lié à l'homme et à la créature; mais son inconditionnalité demeure inviolable. Car la souffrance et la mort nient la prétention du Dieu individuel à l'inconditionnalité (TILLICH 1926a: 11).

\subsection{La victoire finale $d u$ Christ}

C'est le sens même du mystère chrétien qui se trouve par là indiqué. Effectivement, toutes les parties christologiques de la Dogmatique s'appuient sur ce principe. L'essentiel de cette christologie est fort bien résumé dans l'article de 1926 sur «Le concept du démonique et sa signification pour la théologie systématique ". D'emblée, le Christ est situé dans l'histoire des religions, mais il est aussi présenté comme l'ultime assaut contre le caractère démonique de la religion:

De là provient une forte lumière sur l'histoire de la religion et la place du Christ dans cette histoire. Pour le message chrétien aux païens, l'affirmation que dans le Christ les forces démoniques sont vaincues était d'une importance fondamentale. [...] Dans le Christ apparaît cette réalité dans laquelle fondement et profondeur abyssale se trouvent réunis, où l'irruption de la profondeur abyssale du sens se 
déverse dans la parfaite forme du sens, dans la personnalité qui unit la vocation messianique à la volonté de mourir, et qui par là brise en elle-même et triomphe de la force démonique de la religion (TILLICH 1926b : 158-159).

Notons d'abord le contenu de cette pensée sotériologique. Le salut n'est pas simplement l'existence réconciliée, par opposition à l'état antérieur d'aliénation. C'est d'abord et avant tout la victoire finale sur les forces démoniques par l'affirmation inconditionnelle de l'inconditionnalité divine. En d'autres termes, c'est la réalisation parfaite du règne de Dieu par rapport à tous les pouvoirs démoniques. On le voit, la différence est grande avec la christologie présentée par Tillich dans la troisième partie de sa Théologie systématique, où tout se concentre sur la conquête de l'existence aliénée, du péché, par l'être nouveau apparu dans le Christ. Le thème du démonique est alors presque disparu. Ce glissement serait-il sans plus l'effet d'un jugement prudentiel : l'idée du démonique risquant trop d'être mal comprise, mieux valait ne pas l'introduire? Une autre interpréation reste possible, et elle pourrait s'avérer plus féconde. Dans les écrits du Nouveau Testament, le thème du démonique est étroitement lié à celui du règne et du royaume de Dieu, tandis que l'idée de la justification par grâce se réfère elle-même directement au péché plutôt qu'au démonique. Je suggère donc l'hypothèse que la christologie du Tillich allemand s'élabore principalement dans le contexte de l'évangile du royaume, tandis que le Tillich américain mettrait davantage l'accent sur le message paulinien de la justification par la grâce ${ }^{4}$. Tel serait le sens du glissement de l'idée du démonique à celle du péché quant au point de départ de la christologie.

Il s'ensuit une autre différence importante. Dans la Dogmatique de 1925, la christologie prend place dans un contexte historique, au terme d'une histoire théologique des religions; ce contexte est disparu dans la Théologie systématique de 1957, où l'analyse de l'existence a remplacé celle de l'histoire. Autre chose encore. L'idée sotériologique d'un Dieu qui s'anéantit pour triompher du démonique constitue ici, dans la monographie de 1926, une catégorie générale de l'histoire des religions : c'est une troisième voie de salut. Il faut bien voir la signification christologique de cette démarche. D'abord, l'événement Jésus-le-Christ acquiert par là une dimension d'universalité : ce n'est pas un événement purement fortuit, qui surviendrait de façon absolument imprévue, de par le pur effet du bon plaisir divin; tout au contraire, l'avènement du Christ plonge des

$4 \quad$ Voir mon étude de 1992 sur les ramifications de cette problématique au temps du Tillich allemand : "Royaume de Dieu et justification par grâce ", Études Théologiques et Religieuses, 67/4: 495-524. 
racines profondes dans l'histoire de l'humanité, dans l'histoire de la lutte antidémonique.

Mais le caractère unique et gratuit de l'événement n'est pas sacrifié pour autant. Et c'est par la méthode de corrélation que Tillich relie ces deux aspects de l'universalité et de l'unicité. D'une part, l'histoire des religions décrit différentes péripéties d'une lutte constante contre le démonique; elle fait voir aussi quelles sont les conditions requises pour une victoire définitive. Cependant, la religion ne peut par elle-même, de par sa propre dialectique humaine, triompher du démonique. Cela ne peut être que l'effet du salut, l'effet d'une irruption du règne de Dieu dans l'histoire. De même, du point de vue épistémologique, l'histoire des religions indique différentes étapes, toutes relatives, d'un grand combat contre le démonique; mais elle ne peut montrer la victoire définitive du Christ comme avènement du règne de Dieu. Cela ne peut être que l'effet de la révélation, l'effet d'une irruption de la lumière divine dans l'esprit humain. Une telle irruption révélationnelle devient alors principe, point de vue normatif pour la théologie qui, à partir de là, fait retour sur l'histoire des religions. Dans le texte suivant de Tillich, on peut déjà reconnaître ce qu'il appellera plus tard la "méthode de corrélation " : la quête (et la question) du salut d'une part, la réponse de la révélation d'autre part. Cependant, les termes de cette corrélation sont différents de ceux auxquels nous a habitués la Théologie systématique. Il ne s'agit pas ici de la question de l'existence humaine, mais de celle de l'histoire des religions. Et cette question est posée par la présence du démonique, non pas simplement par celle du péché.

Les trois voies pour triompher du démonique dans l'histoire de la religion n'y parviennent pas par elles-mêmes, de par leur propre dialectique. Elles ont une limite intrinsèque, qui ne peut être surmontée que par un acte originaire apparaissant dans l'histoire, que par une irruption de l'inconditionné. Cependant une telle irruption ne peut plus être comprise par une considération dialectique de l'histoire de la religion. Elle n'est accessible que par un acte tout aussi originaire, par une irruption dans l'âme. Mais si elle est ainsi comprise, il est alors possible et nécessaire de montrer en quel sens elle est l'accomplissement du but visé dans l'histoire de la religion, et par conséquent victoire sur le démonique (TILLICH 1926a : 142).

La suite immédiate du texte décrit le cours de l'histoire subséquente dans la voie ouverte par le Christ. Il s'agit de la tradition chrétienne, de l'histoire de l'Église, mais toujours du point de vue du démonique. On doit dire deux choses alors. D'une part, l'Église a toujours veillé à garder la foi en Jésus le Christ pure de toute distortion démonique. C'est là, tout particulièrement, le sens de ses dogmes christologique et trinitaire. Par 
ailleurs, "l'Église elle-même a continuellement succombé devant la force démonique. " Le combat se poursuit donc même si "la foi chrétienne possède la certitude que le démonique est vaincu et qu'est donnée la possibilité de s'approcher du Dieu qui est Dieu en vérité " (TILLICH 1926a : 142).

\subsection{Le sens antidémonique de la sécularisation}

Reste à décrire un dernier épisode dans l'histoire de la lutte contre le démonique. Cette dernière étape se déroule alors parallèlement à l'histoire de la tradition chrétienne. Car le processus s'effectue désormais hors du champ religieux; et pourtant, il s'agit toujours d'une réaction antidémonique. Tillich résume sa pensée en trois phrases lapidaires: " La profanisation s'oppose à toutes les formes religieuses de victoire sur le démonique. Elle aussi constitue une forme de victoire sur le démonique. Mais elle en triomphe en se détachant en même temps du divin" (TILlCH 1926a: 142).

La culture profane procède parallèlement à la religion, mais il importe de noter qu'elle procède aussi de la religion; voilà pourquoi elle poursuit la même lutte antidémonique. La Philosophie de la religion montre bien cette filiation religieuse de la culture autonome (profane ou séculière). Elle constitue la conséquence extrême de l'attitude prophétique ou réformatrice (que Tillich appelle aussi, improprement, "théocratique "), plus ou moins active en toute religion : "En opposition à l'attitude sacramentelle, l'attitude théocratique est orientée vers l'exigence inconditionnée, vers la pure forme " (Tillich 1925a: 91). Elle consiste à "soumettre les formes de la réalité, dans l'agir et dans la connaissance, à la forme inconditionnée, à l'obéissance divine " (TILLICH 1925: 88). C'est en cela précisément qu'elle est antidémonique.

L'attitude prophétique-réformatrice met donc l'accent sur la forme rationnelle de la vérité et de la justice plutôt que sur la substance religieuse et sacrée. Il y a là, par conséquent, la possibilité d'une sortie de la religion: "Si la théocratie n'affirmait dans la loi que la forme de la loi, elle cesserait d'être religion et deviendrait éthique autonome ou métaphysique » (TILlICH 1925a: 91-92). Cette possibilité va se réaliser avec la culture autonome, séculière :

Le mouvement théocratique n'est pas seulement le porteur du développement intra-religieux. C'est de lui également que dépend la possibilité de la culture autonome. En effet, la théocratie et l'autonomie ont ceci de commun qu'elles sont orientées vers la forme. Elles se distinguent pour autant que la théocratie tend vers la forme en tant 
qu'elle est porteuse de la substance inconditionnée, alors que l'autonomie la recherche pour elle-même (TILLICH 1925a : 92).

Dans la monographie de 1926, Tillich décrit plus en détail les différents aspects de la sécularité moderne. Son caractère antireligieux doit s'interpréter au sens d'une lutte contre le démonisme des religions: "Ainsi la philosophie grecque a-t-elle combattu le démonisme des dieux homériques et l'Aufklärung, le démonisme des confesssions chrétiennes.» C'est avec les armes de la forme rationnelle qu'est mené ce combat, et l'on reconnaît alors l'origine divine des valeurs rationnelles. "Mais audelà de la clarté divine, la profondeur divine a été perdue, c'est-à-dire l'inépuisable, ce qui fait irruption, le transcendant inconditionné. [...] Avec les démonismes du passé, qui doivent effectivement être combattus, on a aussi nié les profondeurs divines et créatrices de l'existence" (TILliCH 1926a : 143). C'est comme si l'on avait désamorcé le divin, en lui enlevant sa charge de sacré, qui à tout instant menace de détoner. On reste ainsi avec un Dieu-premier-principe dont on pourra bientôt se passer, qui, de toute façon, tombera de lui-même dans l'insignifiance.

Telle est la version tillichienne de ce qu'on a célébré sous le nom du "désenchantement du monde ». Tillich présente lui-même le phénomène comme une "dédémonisation " (Entdämonisierung) du monde: non plus au sens prophétique et chrétien d'une victoire sur tous les démonismes du monde, mais au sens de l'élimination (ou de l'oubli) de la dimension abyssale et obscure de l'être. Il dira que "la pensée devient bidimensionnelle ». Il n'y a plus que le sujet et l'objet, que la conscience humaine et la nature objective, que la forme et la matière : "La troisième dimension, la hauteur et la profondeur, passe inaperçue : le divin-démonique qui brise la forme, qui gratifie et qui perd " (TILliCH 1926a : 143).

S'opère ainsi un glissement significatif qui va amener finalement un renversement de la situation. Au départ, la protestation contre le démonique provenait d'une soumission à la forme inconditionnée. Mais peu à peu la forme rationnelle n'est plus considérée que dans sa dimension humaine. Elle devient alors un instrument de domination sur la nature d'abord, puis sur la société elle-même. Toute attitude de soumission à une quelconque transcendance divine est supprimée; la finitude s'instaure comme autosuffisante et comme dominatrice du monde :

De cette façon, il devient possible de connaître le monde et de le dominer. Car il ne présente aucune résistance de principe, inconditionnelle. Il est rationnel, même si c'est au prix d'un labeur infini. Il n'y a plus aucun tabou qui retienne la volonté de connaître et de dominer, de se soumettre tout être (TILlICH 1926a : 143-144). 
C'est à ce point précis que s'opère le renversement de la situation. Le démonique revient, en effet, à travers cette nouvelle volonté de puissance propre à la modernité. Et il fait retour avec un pouvoir destructeur accru, à la mesure des nouveaux développements de la rationalité moderne : "Le démonique s'introduit (einbrechen) sans cesse dans la profanité, en tant qu'opposition au divin, en tant que destructeur de la forme, en tant que principe activement négatif» (TILLICH 1926a: 145). On sort donc aisément de la religion, mais il est plus difficile de sortir du démonique. Et avec ce retour du démonique, c'est un nouveau combat qui s'amorce, cette fois sur le sol même de la sécularité moderne.

\section{Les pouvoirs démoniques du temps présent}

Tel est précisément l'objet de la troisième et dernière partie de la monographie de 1926 sur "Le démonique ». Or cette section est beaucoup plus brève que les deux précédentes. Elle se présente sous forme de conclusion et propose un simple schéma. Faut-il croire que Tillich a déjà atteint les limites du temps ou de l'espace dont il dispose? Lui-même laisse plutôt entendre, à deux reprises, que le travail a déjà été fait ailleurs : "La description de cet état de chose et de ses conséquences destructrices a souvent été donnée et ne peut être répétée ici " (TILLICH 1926a : 148). De même, à propos de l'économie capitaliste et de ses méfaits : "Les descriptions de cette destruction des masses et des individus, d'ordre tant spirituel et psychique que corporel, sont si nombreuses et d'une force si irréfutable qu'il n'est pas nécessaire de les répéter ici " (TILliCH 1926a : 150).

Il ne fait pas de doute que Tillich se réfère par là d'abord à ses propres travaux sur le socialisme religieux, tout spécialement à son étude de 1923 sur "Les principes fondamentaux du socialisme religieux". C'est là qu'il met en œuvre, pour la première fois et de façon très élaborée, le concept du démonique. Le socialisme religieux a pour objectif l'état de théonomie que le Nouveau Testament désigne par le symbole du " règne de Dieu». Son combat portera donc sur ce qui s'oppose directement à la théonomie du royaume, c'est-à-dire à la domination du démonique. Dans la partie centrale de son étude, Tillich tentera alors d'identifier les principales manifestations du démonique dans les différentes sphères théoriques et pratiques de notre culture moderne (TILLICH 1923b:178198). En 1926, il présuppose ces analyses de la situation présente, qu'il n'a pas l'intention de répéter. Son travail portera plutôt sur les fondements et les sources du démonique, de même que sur l'histoire du combat antidémonique. Il n'omet pas cependant d'indiquer la troisième dimension du thème: "Les forces démoniques du temps présent »; mais il se 
contente alors de procéder à grands traits, par mode de schéma et de simple rappel. Nous ferons de même à sa suite ${ }^{5}$.

\subsection{Le démon du capitalisme}

Nous avons vu que le nouveau combat contre le démonique prend place maintenant sur le sol de la sécularité moderne. Tillich précise encore : c'est dans la sphère sociale que se trouvent les forces démoniques les plus menaçantes pour notre temps. La théologie devra donc s'aventurer dans le politique; elle devra se faire théologie politique : « Le combat contre les forces démoniques d'une époque devient un devoir religieuxpolitique inévitable. Le politique reçoit la profondeur d'un acte religieux. Le religieux reçoit le caractère concret d'un combat contre " les esprits et. les puissances »" (TILLICH 1926a: 147-148).

Il importe de remonter alors à la racine du mal, qu'on doit chercher en direction du principe même de la modernité, la relation scientifique, objective, aux choses : "Cette attitude, conforme à la nature des choses et appropriée à la relation sujet-objet, est démoniquement déformée par la volonté de domination, qui se saisit d'elle et enlève aux choses leur caractère essentiel et leur propre puissance» (TILliCH 1926a : 148). Par là, non seulement se trouve détruit tout ce qu'il y a de vie et d'autonomie dans les choses, mais la relation vivante avec la nature est elle-même rompue, ce qui ne peut conduire qu'au dépérissement du sujet humain autant que du monde objectif.

C'est dans la sphère pratique de l'organisation sociale que cette domination scientifique et technologique du monde produit ses résultats les plus tangibles. Tillich fait voir d'abord la puissance inégalée de l'économie moderne, capitaliste :

Avec l'aide des moyens que la technique met à sa disposition, l'économie autonome constitue la forme la plus réussie de production de biens qui ait jamais existé. Le mécanisme du marché libre est la machine la plus sophistiquée qu'on puisse concevoir pour l'équilibre de l'offre et de la demande aussi bien que pour l'accroissement constant des besoins et de la satisfaction des besoins (TILLICH 1926a : 149).

Mais justement, cette énorme puissance de l'économie moderne sera celle aussi dont la perversion entraînera les effets les plus désastreux.

5 J'ai déjà fait moi-même une présentation assez substantielle de cette étude de 1923 dans mon "Introduction au Tillich socialiste ", dans Paul TILLICH, Christianisme et socialisme, p. LXIII-LXXVII. 
Tillich parle ici d'« un élément destructeur d'une horrible puissance ", qui exerce son action négative sur les masses et les individus, les atteignant dans tout leur être, tant spirituel et psychique que corporel (TILLICH 1926a : 150).

Apparaissent alors au mieux la signification et la pertinence du concept du démonique. Car le problème soulevé par la force destructrice du capitalisme ne peut être exprimé par de simples catégories morales; il ne peut être résolu non plus par la simple distinction entre l'essence du capitalisme et son usage bon ou mauvais. Non pas que l'essence du capitalisme soit perverse en elle-même, mais la profondeur du mal consiste en ceci que le capitalisme est perverti jusque dans son essence. Et c'est cela précisément que veut exprimer le concept du démonique : "La profondeur du démonique consiste précisément en ceci, qu'en lui sont inséparablement liées la plénitude du sens et la contrariété du sens. De là provient son caractère inévitable, son pouvoir envahissant, devant lequel toute moralisation est condamnée à l'impuissance " (TILlICH 1926a: 150). Voilà bien toute la différence entre une "doctrine sociale", qui ne dépasse pas le niveau d'une éthique sociale, qui se contente de rappeler aux patrons et aux ouvriers leurs devoirs et leurs droits, et une " théologie de la libération" qui, dans les conflits sociaux, au-delà des intérêts divergents des riches et des pauvres, reconnaît l'opposition du divin et du démonique.

\subsection{Le démon du nationalisme}

Dans cette monographie de 1926, Tillich ne se limite pas au démonisme de l'économie capitalisme combattu par le socialisme. Il mentionne encore une autre "grande force démonique du temps présent, le nationalisme". Les potentialités destructrices d'une conscience nationale exacerbée sont apparues manifestement au moment de la Grande Guerre. Elles ont alors été combattues par différents mouvements pacifistes. Ceuxci n'ont pas reconnu cependant la valeur positive du sentiment d'appartenance nationale. Avec sa théorie du démonique comme perversion du sacré, Tillich porte plus avant l'analyse du phénomène :

Les impulsions nationales de l'époque bourgeoise ont été les seules qui avaient et qui ont encore dans une large mesure assez de force pour résister au processus technique de l'économie, qui envahit toute la vie du monde occidental. Elles brisent constamment la pure rationalité. Elles créent une conscience immédiate de consanguinité [...]; elles préservent aussi la conscience du vide total de sens en la remplissant de réalités concrètes. Les choses nationales comportent une inviolabilité sacrée et une dignité cultuelle. - Mais c'est justement par là que commence la démonisation. Avec les forces créatrices sont liées 
les forces destructrices: le mensonge, avec lequel la justice propre d'une nation déforme la véritable image de sa propre réalité et de celle des autres; la violence, qui fait de l'autre peuple un objet dont l'être propre et l'indépendance sont méprisés et écrasés; le meurtre qui au nom du Dieu protecteur de la nation est rehaussé à la dignité de guerre sacrée (TILLICH 1926a : 150).

On peut voir là autant d'allusions à la Guerre, que Tillich a vécue de près comme aumônier militaire. Sans doute, la perversion démonique de la force nationale a-t-elle été démasquée dans les douloureux événements de ces années de guerre. Tillich ajoute cependant cette remarque d'une clairvoyance quasi prophétique : "Ébranlée pour un temps, elle est à présent sur le point de s'affermir à nouveau, autant pour détruire que pour porter. "

Effectivement, quelques années après, il reprend les mêmes analyses et protestations, cette fois contre le démonisme du nationalisme nazi. En 1932, dans ses "Dix thèses sur le National-Socialisme ", il dénonce le protestantisme allemand pour son assujettissement " aux puissances dominantes et à leur démonisme" (thèse 2 ). Son devoir serait plutôt de "garder son caractère prophétique-chrétien, en opposant au paganisme de la croix gammée le christianisme de la croix», et de "témoigner du fait que, sur la croix, le caractère sacré de la nation, de la race, du sang, du pouvoir est rompu et soumis au jugement " (thèse 7). Le protestantisme pourrait ainsi répondre aux justes aspirations « des groupes rassemblés dans le National-Socialisme " et par là même "libérer ce mouvement des démonismes auxquels il est actuellement asservi, qui détruisent le peuple et l'humanité " (thèse 9; TILLICH 1932:3-4).

J'avais, au point de départ, soulevé la question: pouvons-nous, aujourd'hui encore, parler théologiquement du démon? C'est-à-dire : le démon peut-il, aujourd'hui encore, faire partie de la théologie? Au terme de cette étude, je dois répondre : la considération du démonique - sous une forme ou sous une autre, selon une appellation ou l'autre - constitue un élément essentiel de toute théologie, pour la simple raison qu'elle se rapporte au centre même de l'Évangile. Car l'approche du Royaume signifie essentiellement la victoire sur les démons. Et cette victoire s'opère par la croix du Christ, qui est reconcement à toute gloire personnelle pour l'accueil de la puissance même de Dieu. Le défi de la théologie consiste aujourd'hui à produire là-dessus un discours aussi signifiant et percutant qu'a pu l'être en son temps celui de la première communauté chrétienne. C'est la tâche à laquelle s'est attaqué Tillich au début de son enseigne- 
ment universitaire. Il a pour cela démythologisé assez radicalement le discours sur "les démons", vestige d'une autre vision du monde. Et il a tenté de réexprimer pour notre temps la réalité du démonique toujours présente au cœu de l'existence personnelle comme au cœur de la société.

\section{Bibliographie}

ALBRECHT, R. / SCHÜSSLER, W. 1986. Paul Tillich. Sein Werk. Düsseldorf, Patmos, $224 \mathrm{p}$.

DUMAS, Marc. 1997. "Le divin et le démonique d'après la Dogmatique de 1925 », dans Études sur la Dogmatique (1925) de Tillich. Paris, Cerf; Québec, PUL (à paraître au cours de 1997).

PAUCK, Wilhelm \& Marion. 1976. Paul Tillich. His Life $\mathcal{E}$ Thought. New York/San Francisco, Harper \& Row, XII-340 p.

TILLICH, Paul. 1919a. "Sur l'idée d'une théologie de la culture" (trad. N. Grondin), dans La dimension religieuse de la culture. Écrits du premier enseignement (1919-1926). Paris, Cerf; Genève, Labor et Fides; Québec, PUL (CEuvres de Paul Tillich 1), 1990, 29-48.

TILLICH, Paul. 1919b. "Le socialisme : une question pour l'Église ", dans Christianisme et socialisme. Écrits socialistes allemands (1919-1931) (trad. N. Grondin et L. Pelletier), Paris, Cerf; Genève, Labor et Fides; Québec, PUL (GEuvres de Paul Tillich 2), 1992, 11-20.

TILliCH, Paul. 1922. "Masse et esprit. Études de philosophie de la masse », dans Christianisme et socialisme, 47-112.

TILlICH, Paul. 1923a. "Das System der Wissenschaften nach Geganständen und Methoden ", in Philosophische Schriften (G. Wenz ed.), New York, De Gruyter; Berlin, Evangelischer Verlagswerk (Hauptwerke 1), 113-263.

TILLICH, Paul. 1923b. "Les principes fondamentaux du socialisme religieux ", dans Christianisme et socialisme, 169-200.

TILlICH, Paul. 1925a. Philosophie de la Religion (trad. F. Ouellet). Genève, Labor et Fides, 1971, VIII-132 p.

TILLICH, Paul. 1925b. Dogmatik. Marburger Vorlesung von 1925 (W. Schüssler ed.). Düsseldorf, Parmos, 1986, 397 p.

TILlICH, Paul. 1926a. "Le démonique. Un apport à l'interprétation de l'histoire " (trad. M. Thibault), dans La dimension religieuse de la culture, $121-151$. 
TILLICH, Paul. 1926b. "Le concept du démonique et sa signification pour la théologie systématique "( $\operatorname{trad}$. M. Thibault), dans La dimension religieuse de la culture, 153-161.

TILlICH, Paul. 1932. "Dix thèses sur le National-Socialisme", dans Écrits contre les nazis (1932-1935). Paris, Cerf; Genève, Labor et Fides; Québec, PUL (Guvres de Paul Tillich 3), 1994, 1-4. 